\title{
A Key to Improved Ion Core Confinement in the JET Tokamak: Ion Stiffness Mitigation due to Combined Plasma Rotation and Low Magnetic Shear
}

P. Mantica, ${ }^{1}$ C. Angioni,${ }^{2}$ C. Challis,${ }^{3}$ G. Colyer, ${ }^{3}$ L. Frassinetti, ${ }^{4}$ N. Hawkes,${ }^{3}$ T. Johnson, ${ }^{4}$ M. Tsalas, ${ }^{5}$ P. C. deVries,${ }^{6}$ J. Weiland, ${ }^{7}$ B. Baiocchi, ${ }^{1,8}$ M. N. A. Beurskens, ${ }^{3}$ A. C. A. Figueiredo, ${ }^{9}$ C. Giroud,${ }^{3}$ J. Hobirk, ${ }^{2}$ E. Joffrin, ${ }^{10}$ E. Lerche, ${ }^{11}$ V. Naulin, ${ }^{12}$ A. G. Peeters, ${ }^{13}$ A. Salmi, ${ }^{14}$ C. Sozzi, ${ }^{1}$ D. Strintzi, ${ }^{5}$ G. Staebler,${ }^{15}$ T. Tala, ${ }^{16}$ D. Van Eester, ${ }^{11}$ and T. Versloot ${ }^{6}$

${ }^{1}$ Istituto di Fisica del Plasma "P. Caldirola," Associazione Euratom-ENEA-CNR, Milano, Italy

${ }^{2}$ Max-Planck-Institut für Plasmaphysik, EURATOM Association, Garching, Germany

${ }^{3}$ Euratom/CCFE Association, Culham Science Centre, Abingdon, OX14 3DB, United Kingdom

${ }^{4}$ Association EURATOM-VR, Fusion Plasma Physics, EES, KTH, Stockholm, Sweden

${ }^{5}$ Association EURATOM-Hellenic Republic, Athens, Greece

${ }^{6}$ FOM Institute Rijnhuizen, Association EURATOM-FOM, Nieuwegein, The Netherlands

${ }^{7}$ Chalmers University of Technology and Euratom-VR Association, Göteborg Sweden

${ }^{8}$ Università degli Studi di Milano, Department of Physics, Milano, Italy

${ }^{9}$ Associação Euratom-IST, Instituto de Plasmas e Fusão Nuclear, Instituto Superior Técnico, Lisboa, Portugal

${ }^{10}$ Association Euratom-CEA, CEA/IRFM, F-13108 Saint Paul Lez Durance, France

${ }^{11}$ LPP-ERM/KMS, Association Euratom-Belgian State, TEC, B-1000 Brussels, Belgium

${ }^{12}$ Association Euratom-Ris $\phi$ DTU, DK-4000 Roskilde, Denmark

${ }^{13}$ University of Bayreuth, 95440 Bayreuth, Germany

${ }^{14}$ Association EURATOM-Tekes, Aalto University, Department of Applied Physics, Finland

${ }^{15}$ General Atomics, P.O. Box 85608, San Diego, California 92186-5608, USA

${ }^{16}$ Association EURATOM-Tekes, VTT, P.O. Box 1000, FIN-02044 VTT, Finland

(Received 13 May 2011; published 22 September 2011)

\begin{abstract}
New transport experiments on JET indicate that ion stiffness mitigation in the core of a rotating plasma, as described by Mantica et al. [Phys. Rev. Lett. 102, 175002 (2009)] results from the combined effect of high rotational shear and low magnetic shear. The observations have important implications for the understanding of improved ion core confinement in advanced tokamak scenarios. Simulations using quasilinear fluid and gyrofluid models show features of stiffness mitigation, while nonlinear gyrokinetic simulations do not. The JET experiments indicate that advanced tokamak scenarios in future devices will require sufficient rotational shear and the capability of $q$ profile manipulation.
\end{abstract}

DOI: 10.1103/PhysRevLett.107.135004

PACS numbers: 52.25.Fi, 52.35.Ra, 52.55.Fa

Ion temperature gradient (ITG) modes [1-4] are described by theory as featuring a threshold in the inverse ion temperature gradient length $\left(R / L_{T_{i}}=R\left|\nabla T_{i}\right| / T_{i}\right.$, with $R$ the tokamak major radius) above which the ion heat flux $\left(q_{i}\right)$ increases strongly with $R / L_{T_{i}}$. This property leads to a level of stiffness of $T_{i}$ profiles, characterizing how strongly they are tied to the threshold. The role of plasma rotation on threshold and stiffness is of high relevance for predicting the performance of future devices, because the core $T_{i}$ and fusion power achievable for a given $T_{i}$ pedestal depend crucially on threshold and stiffness [5] and future devices are expected to exhibit lower rotation than present devices from which scaling laws are derived.

The role of $E \times B$ flow shear stabilization [6] has been investigated in nonlinear gyrokinetic and fluid simulations [7-10] and found to result in a threshold upshift. From these studies the well-known "Waltz" quenching rule has been derived: $\gamma_{E \times B}=\gamma_{\mathrm{no} E \times B}-\alpha_{E} \omega_{E \times B}$ where $\gamma$ is the instability growth rate, $\omega_{E \times B}$ is the flow shearing rate and $\alpha_{E} \sim 1$ (see [9] for a recent revision). Experimentally, the only study of the impact of rotation on ion threshold and stiffness was performed on JET and reported in [11].
The unexpected result was found that in the plasma core the main effect of rotation is to lower the stiffness rather than increase the threshold, leading to significantly higher $R / L_{T_{i}}$ in rotating plasmas, well above the levels expected by the threshold upshift foreseen by the Waltz rule.

This Letter presents new experimental results on the combined role of rotation and magnetic shear $(s)$ in lowering ion stiffness, using transport tools such as $q_{i}$ scans and $T_{i}$ modulation in plasmas with different safety factor $(q)$ and rotation profiles. The new empirical hypothesis is proposed that the concomitant presence of high rotational shear and low $s$ is the condition for achieving ion stiffness mitigation in tokamaks. The relevance of the results for the improved ion core confinement observed in hybrid regimes [12] or ion internal transport barriers (ITBs) [13] is examined based on JET data. A comparison to state-of-art theory is finally presented, discussing quasilinear fluid and gyrofluid and nonlinear gyrokinetic models.

With reference to the main experiment discussed in [11] [ $q_{i}$ scan at $\rho_{\text {tor }}=0.33$ by varying the localization of ion cyclotron resonance heating (ICRH) power in $\left({ }^{3} \mathrm{He}\right)-D$ minority], reported for the sake of comparison in 


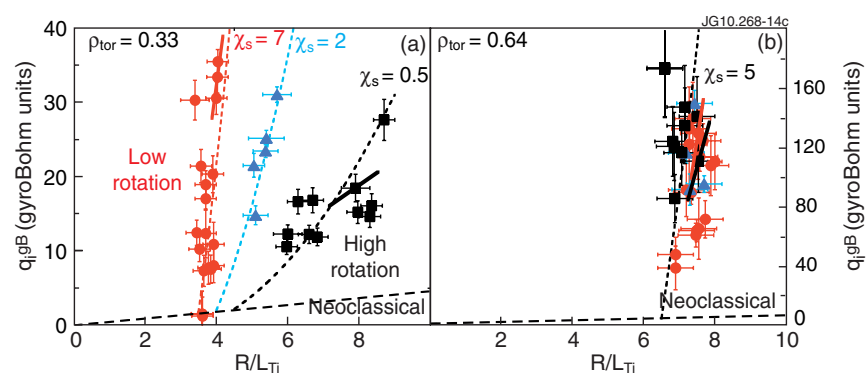

FIG. $1 \quad$ (color online). $\quad q_{i}^{\mathrm{GB}} \quad\left[=q_{i} /\left[\left(\rho_{i} / R\right)^{2} v_{i \text { th }} n_{i} T_{i}\right]\right.$ with $\left.\quad v_{i \text { th }}=\left(T_{i} / m_{i}\right)^{1 / 2}, \quad \rho_{i}=\left(T_{i} m_{i}\right)^{1 / 2} / \mathrm{eB}\right] \quad$ vs $R / L_{T_{i}}$ at (a) $\rho_{\text {tor }}=0.33$, (b) $\rho_{\text {tor }}=0.64$ for similar plasmas with different rotations. @: $1<\omega_{t 0}<2 \times 10^{4} \mathrm{rad} / \mathrm{s} ; \quad \boldsymbol{\Lambda}: 3<\omega_{t 0}<4 \times$ $10^{4} \mathrm{rad} / \mathrm{s} ; \mathbf{\square}: 5<\omega_{t 0}<6 \times 10^{4} \mathrm{rad} / \mathrm{s}$. The dashed black line is indicative of neoclassical transport. The dotted lines represent the critical gradient model (CGM) [11] with different values of $\chi_{s}$.

Fig. 1(a), the analysis of the data at $\rho_{\text {tor }}=0.64$ [Fig. 1(b)] indicates that the stiffness reduction due to rotation (observed at $\rho_{\text {tor }}=0.33$ ) is not present. This is confirmed also by $T_{i}$ modulation data, which yield the slope of the curve as indicated by the two segments. Besides higher values of normalized $q_{i}$ in the outer region, a major difference in plasma parameters between the two regions is the magnetic shear $s=\partial \ln q / \partial \ln \rho$, with $s>1$ at $\rho_{\text {tor }}=0.64$ while $s \sim 0.4-0.6$ at $\rho_{\text {tor }}=0.33$. The rotational shear instead does not show a definite trend with radius. $s$ is determined by EFIT [14] with magnetic, pressure profile, and motional Stark effect (MSE) or polarimeter constraints (statistical error $\sim \pm 0.05$ ).

ICRH $q_{i}$ scans at low and high rotation were carried out in discharges with similar parameters as in Fig. 1's data set $\left(L\right.$ mode, $B_{T}=3.36 \mathrm{~T}, I_{p}=1.8 \mathrm{MA}, n_{e 0}=$ $\left.3-4 \times 10^{19} \mathrm{~m}^{-3}\right)$, but in which the $q$ profile, and hence $s$ and $s / q$, were varied by lower hybrid preheat, plasma current $\left(I_{p}\right)$ ramp-up and ramp-down (1.8 to $3 \mathrm{MA}$ in $3.5 \mathrm{~s}), I_{p}$ overshoot [12]. $s$ was varied between 0.05 and $0.8(0.02<s / q<0.5)$ at $\rho_{\text {tor }}=0.33$ and between 0.75 and $1.45(0.25<s / q<0.7)$ at $\rho_{\text {tor }}=0.64$. The aim was to investigate if a low $s$ value is indeed a concomitant requirement for stiffness reduction due to rotation. Ion threshold and stiffness have been identified as in [11] by placing on- and off-axis 3 MW of ICRH power in $\left({ }^{3} \mathrm{He}\right)-D$ minority at concentrations $n_{3} \mathrm{He} / n_{e} \sim 6 \%-8 \%$ and measuring $R / L_{T_{i}}$ with active charge exchange spectroscopy. $T_{i}$ modulation was performed on top. In the high rotation discharges, up to $11 \mathrm{MW}$ of coinjected neutral-beam (NBI) power was applied. Figure 2(a) shows the time evolution of the $q$ profile in a low rotation shot with $I_{p}$ ramp-up and off-axis ICRH. $s$ and $s / q$ at $\rho_{\text {tor }}=0.33$ and 0.64 are shown vs time in Fig. 2(b). In low NBI discharges, positioning ICRH off-axis implies that the actual $R / L_{T_{i}}$ at $\rho_{\text {tor }}=0.33$ is a measure of threshold, since $q_{i} \sim 0$. Therefore, the time evolution of $R / L_{T_{i}}$ directly yields the dependence of threshold on $s / q$, which is expected from linear theory to be
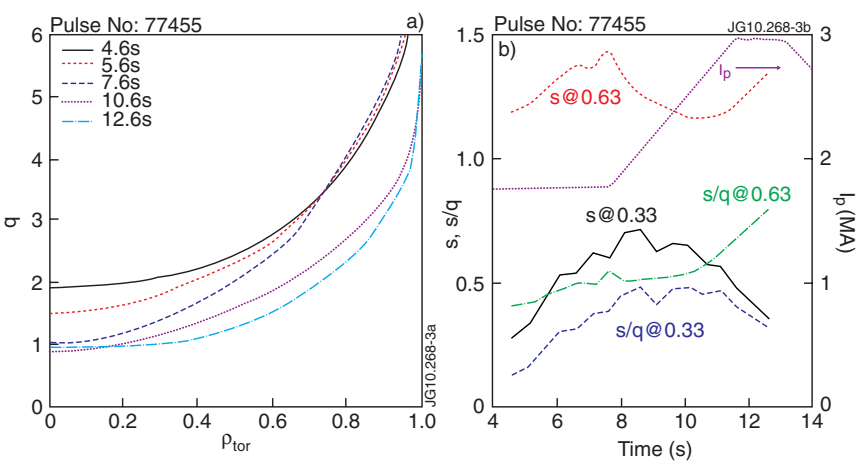

FIG. 2 (color online). (a) time evolution of MSE $q$ profile for pulse 77455 ; (b) $I_{p}, s, s / q$ vs time at two radii.

stabilizing. Figure 3 shows $R / L_{T_{i}}$ vs time at $\rho_{\text {tor }}=0.33$ for the low and high rotation $I_{p}$ ramp-up shots, and the linear ITG threshold (similar for both shots) predicted by an analytical formula proposed in [15]

$$
R / L_{T_{i}}^{\mathrm{ITG}}=\frac{4}{3}\left(1+\frac{T_{i}}{T_{e}}\right)\left(1+2 \frac{s}{q}\right) \text { for } \frac{R}{L_{n}}<2\left(1+\frac{T_{i}}{T_{e}}\right) .
$$

The increase in measured threshold with time following the increase of $s / q$ is in good agreement with the expected dependence from Eq. (1). In spite of such threshold increase, it is remarkable that the time behavior of $R / L_{T_{i}}$ in the high rotation shot is opposite, with $R / L_{T_{i}}$ lying 3 times above threshold in the early phase and dropping to a factor 1.3 of threshold at late times. At low rotation instead also the on-axis ICRH case (not shown, but virtually identical to the off-axis case) due to high stiffness keeps close to threshold with $R / L_{T_{i}}$ increasing with time. This observation is a beautiful confirmation of the fact that at high rotation the core $T_{i}$ dynamics is completely dominated by stiffness, and the stiffness reduction is more pronounced when the $q$ profile is flatter (i.e., at early times). At outer radii, where stiffness is high irrespective of rotation, $R / L_{T_{i}}$ both at low and high rotation keeps close to 7.5. The transport changes in Fig. 3 are accompanied by consistent changes in turbulence measured by correlation reflectometry [16], as discussed in [17].

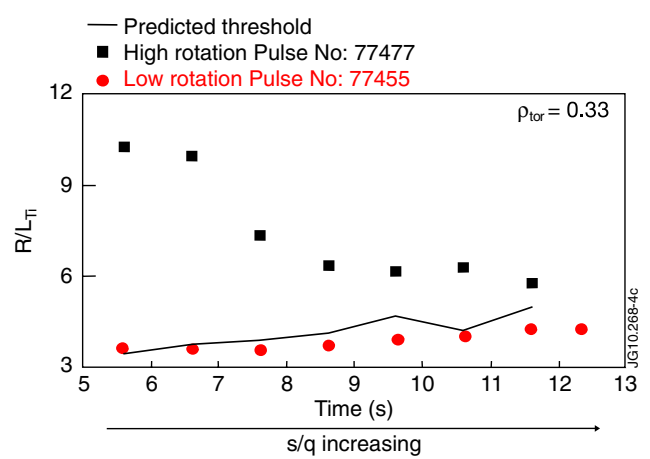

FIG. 3 (color online). Experimental $R / L_{T_{i}}$ at $\rho_{\text {tor }}=0.33$ vs time at low (red circles) and high (black squares) rotation for the $I_{p}$ ramp-up case, and ITG threshold predicted with Eq. (1). 


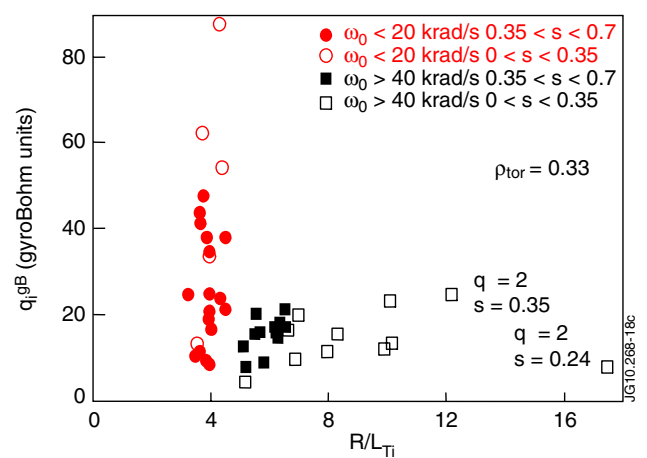

FIG. 4 (color online). $\quad q_{i}{ }^{\mathrm{GB}}$ vs $R / L_{T_{i}}$ at $\rho_{\text {tor }}=0.33$ for similar plasmas with different rotation and $s$ values.

Similar observations are made with the other schemes for $q$ profile variation. The data are summarized in Fig. 4. $q_{i}$ values are estimated by the PION ICRH code [18], $s$ is from MSE. Low rotation data show high stiffness irrespective of $s$, while at high rotation the stiffness reduction is larger at low $s$, allowing $R / L_{T_{i}}$ up to 10 even at low $q_{i}{ }^{\mathrm{GB}}$.

Very high $R / L_{T_{i}}(>10)$ is seen at high rotations when the $q=2$ surface is at low $s$, while at higher $s$ it does not lead to $R / L_{T_{i}}$ increase. This is in line with observations of the beneficial role of low order rationals near $s=0$ on turbulent transport [19-22], for which a theoretical basis was proposed in [23]. Such effect of rationals appears as a different phenomenon, which adds to the stiffness mitigation discussed in this Letter. In fact, the effect of rationals is reported both on ions and electrons [19-22] and also in the absence of rotation [19,22], while the stiffness mitigation is only observed on ions and strictly linked to rotation.

The evidence of ion stiffness reduction due to rotation and low $s$ has profound implications on the interpretation of improved ion core confinement in hybrid plasmas or ion ITBs, proposing an alternative paradigm to the usual one based on $E \times B$ flow shear threshold up-shift. As a matter of fact, both regimes are experimentally observed in conditions of strong rotation and are lost in the absence of rotation [22,24]. Also, an important role of $q$ profile manipulation is recognized experimentally (e.g., $[12,13])$. In [25] the role of both rotation and low $s$ was already deduced from JET ITB experimental data. Figure 5 (inset) shows typical JET $q$ profiles in 4 regimes: standard $H$ mode with fully diffused current, hybrid, optimized shear (OS) and negative shear (NS) ITBs. Both hybrids and ITBs have core regions of very low $s$. Their position in the $q_{i}^{\mathrm{GB}}$ vs $R / L_{T_{i}}$ plot at $\rho_{\text {tor }}=0.33$ (Fig. 5) shows that the data populate uniformly the region of high $R / L_{T_{i}}$ and low $q_{i}{ }^{\mathrm{GB}}$. Some considerations can be made to discriminate if this behavior is mainly due to stiffness or threshold.

In the core of hybrids the linear threshold was found between 3.5 and 5 using GS2 [26], well below the actual $R / L_{T_{i}} . \quad \omega_{E \times B} \sim 3-4 \times 10^{4} \mathrm{~s}^{-1}$, yielding threshold upshifts $\sim 1$. The profiles then lie well above threshold even at small $q_{i}^{\mathrm{GB}}$, indicating low stiffness. This was also confirmed by NBI $T_{i}$ modulation (not shown). $R / L_{T_{i}}$ vs $s$ at

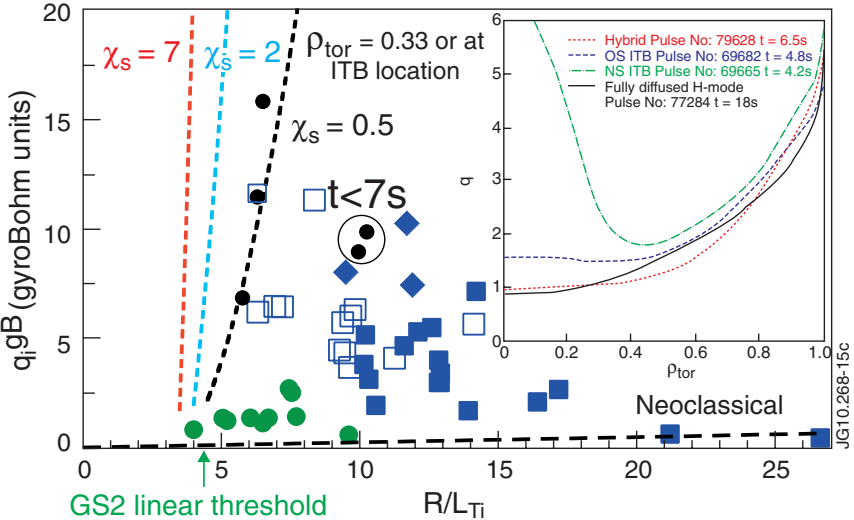

FIG. 5 (color online). $\quad q_{i}{ }^{\mathrm{GB}}$ vs $R / L_{T_{i}}$ at $\rho_{\text {tor }}=0.33$ for a set of hybrids (green or gray circles), ion ITBs at trigger time (blue open squares) and fully developed (blue full squares, diamonds with large ICRH fraction and reduced rotation). The data of Fig. 1(a) are indicated by CGM fits (dashed lines). Black circles are from pulse 77477 at different times (cf. Fig. 3). Neoclassical level and GS2 linear threshold for a hybrid are shown. The inset shows $q$ profiles from MSE for the four scenarios.

low and high rotation is plotted in Fig. 6 from a JET $H$ mode and hybrid database. The scatter is due to the range in parameters in the database, in first place $q_{i}$. Still, it is remarkable that the two clouds clearly separate at low $s$, with larger $R / L_{T_{i}}$ at high rotation. This suggests that stiffness mitigation in the broad low $s$ region is at the origin of the improved core ion confinement. The dependence on $s$ is also one reason (together with different deposition profiles) why not much effect is seen in fully diffused $H$ modes when ICRH power is substituted to NBI power [27].

For ITBs, the profiles at trigger yield $\omega_{E \times B} \sim$ $1-2 \times 10^{4} \mathrm{~s}^{-1}$, not producing large threshold up-shift. The data lie in Fig. 5 in between the rotating shots of Fig. 1(a) and the neoclassical level. In fully developed ITBs, however, the ITB itself generates a large $\nabla \omega_{t}$ at the ITB radius, with $\omega_{E \times B} \sim 7-8 \times 10^{4} \mathrm{~s}^{-1}$, inducing significant threshold up-shifts. It is then difficult to separate the role of threshold and stiffness. $T_{i}$ modulation has been

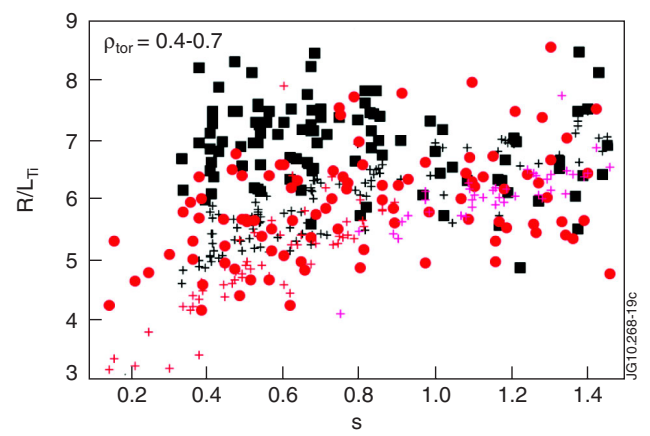

FIG. 6 (color online). $\quad R / L_{T_{i}}$ vs $s$ at $\rho_{\text {tor }}=0.4-0.7$ at low [red circles, $\left.\nabla \omega_{t}<50 \mathrm{krad} /(\mathrm{ms})\right]$ and high [black squares, $\nabla \omega_{t}>$ $130 \mathrm{krad} /(\mathrm{ms})]$ rotation from a hybrid and $H$-mode JET database. $s$ is from EFIT with polarimeter. Crosses are threshold values after Eq. (1). 


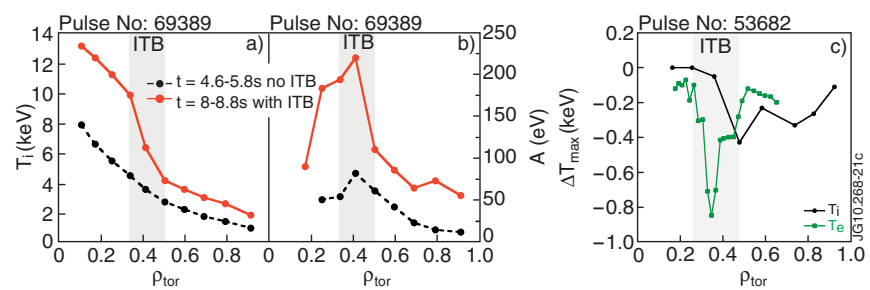

FIG. 7 (color online). (a) $T_{i}$, (b) amplitudes for an ITB shot before trigger (black dashed line) and at full strength (red full line). (c) Ion and electron cold pulse maximum amplitude.

performed using ${ }^{3} \mathrm{He}$ ICRH modulation. $T_{i}$ and amplitude profiles with and without ITB are compared in Figs. 7(a) and 7(b). The ITB acts as a layer of low incremental diffusivity, with sharp variation of amplitudes, indicating a low slope of the $q_{i}$ vs $R / L_{T_{i}}$ plot. These observations, reported also for electrons in [28], are not consistent with $T_{i}$ profiles tight to a high threshold by high stiffness, but rather with either transport above critical with low stiffness, or with subcritical transport. The second hypothesis must be discarded because in most cases ITBs and hybrids are found well above neoclassical (Fig. 5). We conclude that also in ion ITBs the pattern of decreasing stiffness plays a major role, with the threshold up-shift intervening in a nonlinear feedback while the ITB develops. Such a picture for ions is different from that for electrons proposed in [28], with electron ITBs as subcritical regions in the presence of stiff electron transport. The difference is confirmed by the behavior of electron and ion cold pulses when they meet the ITB propagating from edge [Fig. 7(c)]. The electron cold pulse grows significantly inside the ITB [28], due to redestabilization of stiff turbulent transport [29]. For ions, with low stiffness in the ITB, the cold pulse growth cannot take place but strong damping is seen.
While in ITB plasmas the $H$ factor $\sim 1.5$ is all due to large core gradients, in hybrids enhanced pedestal and neoclassical tearing mode (NTM) stabilization also contribute to the $H$ factor. It was shown statistically [30] that the contribution of pedestal to total energy is $20 \%-40 \%$ both in $H$ modes and hybrids and that the $H$ factor up to 1.5 in hybrids is due in equal parts to core and pedestal improvement, with the core part mainly in the ion channel. These findings are consistent with our estimate that an increase in $R / L_{T_{i}}$ from 6 to 10 due to stiffness mitigation in a region up to $\rho_{\text {tor }}=0.6$ at fixed pedestal leads to $\Delta H \sim 0.2$. With regard to NTMs, experiments show [31] that strong NTM onset leads to an abrupt drop of the $H$ factor to $\sim 1$, with a deterioration of $T_{i}$ profile mainly caused by the braking of plasma rotation. This does not contradict the fact that, when NTMs are weak or absent, the core transport dynamics discussed above are at play.

Finally, we address the state of the art of theory predictions on the effect of rotation on ion transport. In widely used quasilinear transport models, such as WEILAND [32] or GLF23 [33], which apply the Waltz rule on a given and restricted choice of spectral wave numbers, rotation introduces only a small threshold up-shift and not a change in slope. The results by the WEILAND model for a shot with $s=0.57$ are shown in Fig. 8(a) without and with rotation $\left[\gamma_{E}=\omega_{E \times B} / c_{s} / a \sim 0.1\right.$, with $\left.c_{s}=\left(T_{e} / m_{i}\right)^{1 / 2}\right]$. On the other hand, the more recent gyrofluid TGLF model [34], which makes use of full spectra, indicates a change in slope [Fig. 8(b)], in particular, in the region of the knee, at the transition between fully developed turbulence and zonal flows. The simulations scan $R / L_{T_{i}}$ and $R / L_{T_{e}}$ in a prescribed ratio (from experiment) without and with rotation. This effect may be due to differential suppression of turbulence at various wavelengths, with more suppression of the low stiffer ones. The change in stiffness with rotation is larger at low $s$ [Fig. 8(d)] as in experiments. The WEILAND model has been modified to include the dependence
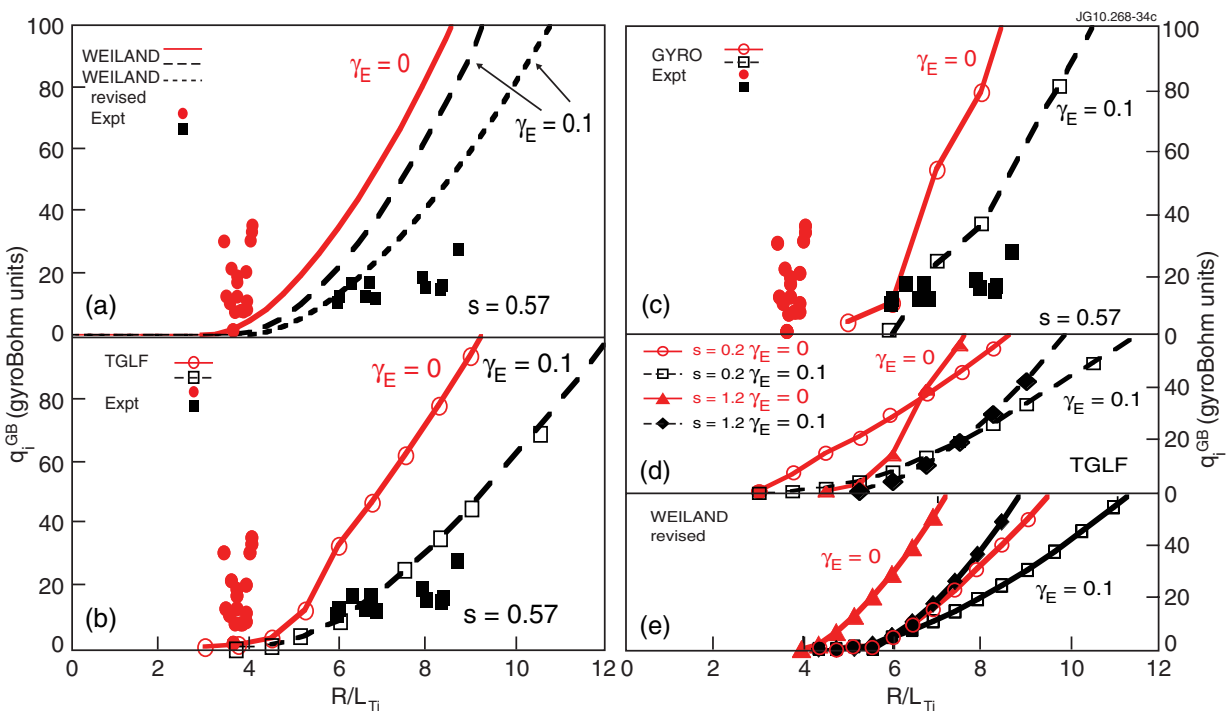

FIG. 8 (color online). $\quad q_{i}{ }^{\mathrm{GB}}$ vs $R / L_{T_{i}}$ at $\rho_{\text {tor }}=0.33$ without and with rotation from (a) WEILAND, (b) TGLF, and (c) GYRO simulations with $s=0.57$ compared with the data of Fig. 1(a). (d) TGLF and (e) revised WEILAND [legend as in (d)] simulations with $s=0.2$ and 1.2 
of the fastest growing mode number on rotation [35], obtaining results very similar to TGLF [Figs. 8(a) and 8(e)].

To verify these results, nonlinear flux-tube electrostatic gyrokinetic simulations using GYRO [36] have been made [Fig. 8(c)], with background rotation and electron-ion collisions, for the same parameters as with TGLF. The box size is $62 \times 222 \rho_{s}\left(\rho_{s}=\left(T_{e} m_{i}\right)^{1 / 2} / \mathrm{eB}\right)$ with 64 toroidal modes from $k_{y} \rho_{s}=0.028$ to $k_{y} \rho_{s}=1.78$ and with a minimum $k_{x} \rho_{s}=0.1$ and $\max k_{x} \rho_{s}=6.47$ corresponding to a radial resolution $\delta x / \rho_{s}=0.24$. The radial box size is $\Delta r / a=$ 0.125 . At low $R / L_{T_{i}}$, very large $n=0$ (zonal) electrostatic potential fluctuations are found, which strongly reduce the transport and obligate us to perform simulations exceeding $1000 a / c_{s}$. The same results are obtained with double radial box size keeping the same radial resolution. We note that runs with a reduced number of toroidal modes do not deliver the same strength of zonal flows. With 64 modes, due to the large zonal flows, turbulent transport is rapidly quenched to zero near threshold and there is no hint of stiffness mitigation at high rotation, but basically only a threshold up-shift. Similar results were obtained with GKW [37] as reported in [17] and recently with GS2 on a different case [ [38], Fig. 3(a)]. Global simulations did not yield significant changes at this inner radius. Since the mechanisms mentioned above as a possible origin of the stiffness reduction in quasilinear models are also included, and with most resolved treatment, in nonlinear gyrokinetic models, we have to admit that presently the impact of rotation on ion stiffness remains an open issue on the theory side. It deserves further work because, although numerically challenging, the marginality region is the operating domain of fusion devices. We also note that none of the models in Fig. 8 in fact reproduce correctly the low $R / L_{T_{i}}$ of the low rotation data, either due to not high enough stiffness or to the presence of a nonlinear (Dimits) threshold up-shift. This is also an open issue.

In summary, JET experiments show that ion stiffness is reduced by low $s$ and high rotation. This indicates that advanced tokamak scenarios in future devices will require sufficient rotational shear and the capability of $q$ profile manipulation.

Nonlinear gyrokinetic simulations were performed on the parallel server Power 6 (Vip) of the IPP-MPG Rechenzentrum Garching, Germany. This work was supported by EURATOM and carried out within the framework of EFDA. The views and opinions expressed herein do not necessarily reflect those of the European Commission. This work was done under the JET-EFDA workprogramme [39].

[1] N. Mattor et al., Phys. Fluids 31, 1180 (1988).

[2] F. Romanelli et al., Phys. Fluids B 1, 1018 (1989).

[3] J. W. Connor and H. R. Wilson, Plasma Phys. Controlled Fusion 36, 719 (1994).

[4] M. Kotschenreuther et al., Phys. Plasmas 2, 2381 (1995).

[5] B. Baiocchi et al., Plasma Phys. Controlled Fusion (to be published).
[6] H. Biglari et al., Phys. Fluids B 2, 1 (1990).

[7] J.E. Kinsey et al., Phys. Plasmas 12, 062302 (2005).

[8] R. E. Waltz et al., Phys. Plasmas 1, 2229 (1994).

[9] J. E. Kinsey et al., Phys. Plasmas 14, 102306 (2007).

[10] X. Garbet et al., Phys. Plasmas 9, 3893 (2002).

[11] P. Mantica et al., Phys. Rev. Lett. 102, 175002 (2009).

[12] J. Hobirk et al., Plasma Phys. Controlled Fusion (to be published).

[13] R. C. Wolf, Plasma Phys. Controlled Fusion 45, R1 (2003).

[14] L. L. Lao et al., Fusion Sci. Technol. 48, 968 (2005) [http://www.new.ans.org/pubs/journals/fst/a_1052].

[15] S. C. Guo and F. Romanelli, Phys. Fluids B 5, 520 (1993).

[16] A. C. A. Figueiredo et al., in Proceedings of the 36th EPS Conference, Sofia, 2009, Europhysics Conference Abstracts, Vol. 33E, P-2.167, http://epsppd.epfl.ch/Sofia/ pdf/P2_167.pdf.

[17] P. Mantica et al., in Proceedings of the 23rd IAEA Fusion Energy Conference, Daejeon, 2010 (IAEA, Vienna, 2010), EXC/9-2 [http://www-pub.iaea.org/mtcd/meetings/PDFplus/ 2010/cn180/cn180_papers/exc_9-2.pdf].

[18] L.-G. Eriksson et al., Nucl. Fusion 33, 1037 (1993).

[19] N. J. Lopes Cardozo et al., Plasma Phys. Controlled Fusion 39, B303 (1997).

[20] M. E. Austin et al., Phys. Plasmas 13, 082502 (2006).

[21] E. Joffrin et al., Nucl. Fusion 42, 235 (2002).

[22] P. C. de Vries et al., Nucl. Fusion 49, 075007 (2009).

[23] R. E. Waltz et al., Phys. Plasmas 13, 052301 (2006).

[24] P. A. Politzer et al., Nucl. Fusion 48, 075001 (2008).

[25] T. Tala et al., Plasma Phys. Controlled Fusion 43, 507 (2001).

[26] M. Kotschenreuther et al., Comput. Phys. Commun. 88, 128 (1995).

[27] T. W. Versloot et al., Nucl. Fusion (to be published).

[28] P. Mantica et al., Phys. Rev. Lett. 96, 095002 (2006).

[29] A. Casati et al., Phys. Plasmas 14, 092303 (2007).

[30] L. Frassinetti et al., in Proceedings of the 37th EPS Conference, Dublin, 2010, Europhysics Conference Abstracts, Vol. 34A, P-1.1031, http://ocs.ciemat.es/ EPS2010PAP/pdf/P1.1031.pdf.

[31] C.D. Challis et al., in Proceedings of the 36th EPS Conference, Sofia, 2009, Europhysics Conference Abstracts, Vol. 33E, P-5.172, http://epsppd.epfl.ch/Sofia/ pdf/P5_172.pdf.

[32] J. Weiland, Collective Modes in Inhomogeneous Plasmas (IOP, Bristol, 2000).

[33] R. E. Waltz et al., Phys. Plasmas 4, 2482 (1997).

[34] G. M. Staebler et al., Phys. Plasmas 12, 102508 (2005).

[35] J. Weiland et al., in Proceedings of 38th EPS Conference, Strasbourg, 2011, Europhysics Conference Abstracts, P5.130, http://ocs.ciemat.es/EPS2011PAP/pdf/P5.130.pdf (to be published).

[36] J. Candy and R. E. Waltz, J. Comput. Phys. 186, 545 (2003).

[37] A. G. Peeters et al., Comput. Phys. Commun. 180, 2650 (2009).

[38] E. G. Highcock et al., Phys. Rev. Lett. 105, 215003 (2010).

[39] F. Romanelli et al., in Fusion Energy 2010: Proceedings of the 23rd International Conference, Daejeon, 2010 [International Atomic Energy Agency (IAEA), Vienna, 2010] [http://www-pub.iaea.org/mtcd/meetings/PDFplus/ 2010/cn180/cn180_papers/ov_1-3.pdf]. (All the members of the JET-EFDA Collaboration appear in the appendix of this paper.) 